\title{
Overview of Mid-Infrared Observatories
}

\author{
Ewine F. van Dishoeck \\ Leiden Observatory, P.O. Box 9513, NL-2300 RA Leiden, The \\ Netherlands
}

\begin{abstract}
A brief overview of mid-infrared science and future groundand space-based facilities at mid-infrared and submillimeter wavelengths is given.
\end{abstract}

\section{Introduction and mid-infrared features}

The formation of stars and planetary systems takes place deep inside clouds of gas and dust, often obscured by hundreds of magnitudes of extinction. At high redshifts, the assembly of galaxies and the first bursts of star formation are accompanied by large amounts of dust. In order to penetrate these dusty regions, observations at long wavelengths (mid-infrared, submillimeter) are essential.

The ISO satellite has opened up the mid-infrared wavelength region, and has shown the wealth of features and their diagnostic value (Cox \& Kessler 1999). The continuum is dominated by thermal emission from warm dust with $T_{\text {dust }} \approx 100-300 \mathrm{~K}$. The lines range from atomic and ionic recombination- and fine-structure lines, to PAH features, solid-state bands, and gas-phase molecular lines, each of which probe different physical characteristics (see van Dishoeck 2000, van Dishoeck \& Tielens 2001 for overviews).

Atomic and ionic fine-structure lines: Fine-structure transitions within the lowest electronic term of most astrophysically relevant atoms and ions occur in the mid- and far-infrared wavelength range. Important examples are the [Ar II] 7.0, [Ne II] 12.8, [Ne V] 14.3, [Ne III] 15.6, [S I] 25.2, [O IV] 25.9, [O I] 63, [O III] 88 and [C II] $158 \mu \mathrm{m}$ lines, but even higher ionization stages ("coronal" lines) can be observed. These features are used as probes of the ionizing source, especially the hardness of the radiation field (e.g., starbursts vs. AGN), and as diagnostics of heating mechanisms, in particular shock- vs. photon-heating. The strongest lines can also be used to trace the kinematics of gas in obscured regions.

PAHs: The C-C and C-H stretching and bending modes of polycyclic aromatic hydrocarbons (PAHs) at $6.2,7.7,8.6,11.3, \ldots \mu \mathrm{m}$ often dominate the midinfrared emission. More recently, new features at $16.2 \mu \mathrm{m}$ and longer wavelengths have been found in ISO-SWS spectra, which contain diagnostic information about the type of species involved. The PAH features trace the presence of stellar ultraviolet radiation.

$\mathrm{H}_{2}$ and $\mathrm{HD}$ pure rotational lines: The lowest transitions of the most abundant molecule in the universe, $\mathrm{H}_{2}$, occur at mid-infrared wavelengths $(J=2-0 \mathrm{~S}(0)$ at $28.22 \mu \mathrm{m}, J=3-1 \mathrm{~S}(1)$ at $17.03 \mu \mathrm{m})$. These lines provide a direct measure of the mass and temperature of the bulk of warm molecular gas at $T=50-200 \mathrm{~K}$. 
The lines of the heavier isotope, $\mathrm{HD}$, occur at $112,56, \ldots \mu \mathrm{m}$. In combination with $\mathrm{H}_{2}$, they can be used to determine the $[\mathrm{D}] /[\mathrm{H}]$ ratio in star-forming regions.

Solid-state vibrational bands: The characteristic vibrational bands of ices, silicates and oxides occur uniquely at mid-infrared wavelengths. The $\mathrm{Si}-\mathrm{O}$ stretching and bending bands of amorphous silicates at 9.7 and $18 \mu \mathrm{m}$ are well known, but features of crystalline silicates and oxides at longer wavelengths have been discovered only recently by ISO in circumstellar matter around young and old stars (Waelkens et al. 2000). A wealth of vibrational ice bands occurs between 3 and $16 \mu \mathrm{m}$, whereas weaker librational ice modes occur at longer wavelengths out to $100 \mu \mathrm{m}$. The line shapes and abundances of the solid-state species are sensitive diagnostics of heating events and the temperature history of the region.

Gas-phase vibrational bands: Fundamental vibrational transitions of important organic molecules such as $\mathrm{CH}_{4}, \mathrm{C}_{2} \mathrm{H}_{2}, \mathrm{HCN}$ and $\mathrm{CO}_{2}$ occur at $7.7,13.7,14.0$ and $15.0 \mu \mathrm{m}$, whereas the bending mode of $\mathrm{H}_{2} \mathrm{O}$ lies at $6.0 \mu \mathrm{m}$. At longer wavelengths, the pure rotational transitions of $\mathrm{H}_{2} \mathrm{O}, \mathrm{OH}, \mathrm{CO}$ and $\mathrm{HCN}$ become prominent in far-infrared spectra of young stellar objects and AGB stars. The excitation of these species provides valuable constraints on temperature and density.

\section{Future observatories}

Because of the large potential gains in sensitivity and spatial resolution, a wealth of new observatories operating from mid-infrared to submillimeter wavelength are being constructed or planned. In rough chronological order, the most relevant facilities are:

SIRTF: The Space InfraRed Telescope Facility is a $85 \mathrm{~cm}$ cooled telescope to be launched by NASA in mid-2002. It has two cameras covering the $3-180 \mu \mathrm{m}$ range, and one spectrometer covering 5-40 $\mu \mathrm{m}$ at $R=\lambda / \Delta \lambda=60-120$ and $10-38$ $\mu \mathrm{m}$ at $R=600$. See http://sirtf.jpl.nasa.gov/.

SOFIA: The Stratospheric Observatory For Infrared Astronomy, a joint USGerman project, is a $2.5 \mathrm{~m}$ telescope in a B747 airplane starting flights in 2003 . Although its sensitivity will be lower than that of SIRTF because of the higher temperature, it will have a phenomenal set of state-of-the-art instrumentation (cameras, low- and high-resolution spectrometers) covering the full $2-500 \mu \mathrm{m}$ range at $R=3$ to $10^{6}$. See http://www.sofia.nasa.gov/.

FIRST: The Far-InfraRed and Submillimeter Telescope, an ESA cornerstone mission in collaboration with NASA, is a $3.5 \mathrm{~m}$ telescope to be launched in 2007 . It covers the $\sim 80-500 \mu \mathrm{m}$ wavelength range with three instruments, including an imaging spectrometer with $R \approx 3000$ from $80-200 \mu \mathrm{m}$ and heterodyne receivers with $R>10^{6}$ from 200-500 $\mu \mathrm{m}$. See http://astro.estec.esa.nl/SA-general /Projects/First/.

NGST: The Next Generation Space Telescope, the 8-m successor of the Hubble Space Telescope, is a joint NASA-ESA-CSA project optimized for near-infrared wavelengths with a planned launch in 2009. The recommended suite of instruments includes a mid-infrared camera/spectrometer, with a sensitivity surpassing that of previous facilities by orders of magnitude and with a spatial resolu- 
tion that is a factor of 10 higher than that of SIRTF. See http://ngst.gsfc. nasa.gov/ and http://astro.estec.esa.nl/SA-general/Projects/NGST/.

$A L M A$ : The Atacama Large Millimeter Array is a joint project between the US and Europe to build an array of $64 \times 12 \mathrm{~m}$ telescopes on the Chajnantor 'altiplano' in Chile to be completed by $\sim 2010$. Japan may also join the project. It will be equipped with heterodyne receivers covering the 30-900 $\mathrm{GHz}$ range at $R>10^{6}$. Its observing speed will surpass that of existing interferometers by up to a factor of $10^{4}$. See http: //www.alma.nrao.edu/ and http://www.eso.org/projects/ alma/.

TPF/DARWIN: The US and Europe are developing plans for a mid-infrared $(\sim 5-20 \mu \mathrm{m})$ interferometer in space, to be launched after 2012 . The main goal of this mission is to image extra-solar Earth-like planets through nulling interferometry and to measure the composition of their atmospheres. A wealth of additional science will be possible as well. See http://tpf.jpl.nasa.gov/ and http://astro.estec.esa.nl/SA-general/Projects/IRSI/.

Ground-based optical telescopes: Several of the existing $8-10 \mathrm{~m}$ class optical telescopes are equipped with mid-infrared (8-25 $\mu \mathrm{m})$ cameras and spectrometers. Although ground-based facilities suffer heavily from atmospheric interference and high thermal backgrounds, they provide state-of-the-art instrumentation and higher spectral resolution than possible from space. Future $30-100 \mathrm{~m}$ class optical telescopes will be powerful complements to NGST for mid-infrared imaging and spectroscopy of bright sources at higher spatial and spectral resolution.

\section{References}

Cox, P. \& Kessler, M.F., eds. 1999, The Universe as seen by ISO, ESA SP-427 (Noordwijk: ESTEC)

van Dishoeck, E.F. 2000, in NGST Science \& Technology Exposition, eds. E.P. Smith \& K. Long (ASP), 85

van Dishoeck, E.F. \& Tielens, A.G.G.M. 2001, to appear in The Century of Space Science, eds. J. Bleeker et al. (Kluwer)

Waelkens, C., Malfait, K., \& Waters, L.B.F.M. 2000, in Astrochemistry: From Molecular Clouds to Planetary Systems, IAU Symposium 197, eds. Y.C. Minh \& E.F. van Dishoeck (ASP), 435 